\title{
IMPACTO DA DOR LOMBAR EM GESTANTES E PRINCIPAIS INTERVENÇÕES TERAPÊUTICAS: UMA REVISÃO DA LITERATURA
}

\author{
IMPACT OF LOW BACK PAIN ON PREGNANT WOMEN AND MAIN THERAPEUTIC INTERVENTIONS: A \\ LITERATURE REVIEW
}

Ruana Glicya Lima Silva ${ }^{1}$, Alice Anny Diniz Rocha ${ }^{1}$, Jessica Bruna Florêncio e Silva ${ }^{1}$, Wiara Milleny Roque Linhares ${ }^{1}$, Elanny Mirelle da Costa ${ }^{1}$, Joelma Gomes da Silva

${ }^{1}$ Acadêmicos da Faculdade Nova Esperança de Mossoró - FACENE/RN

\section{Info}

Recebido: 07/2020

Publicado: $11 / 2021$

DOI: 10.37951/2358-260X.2021v8i2.5913

ISSN: 2358-260X

Palavras-Chave

Dor lombar. Condutas terapêuticas.

Exercícios físicos. Gestantes. Qualidade de vida.

Keywords:

Low back pain. Therapeutic approaches. Physical exercises. Pregnant women. Quality of life.

\section{Resumo}

0 período gestacional é marcado por diversas mudanças morfológicas, biomecânicas e fisiológicas, apesar de ser considerado um evento normal diversos desafios são apresentados a gestante principalmente a lombalgia. Esse estudo trata-se de uma revisão integrativa da literatura que busca realizar um levantamento acerca dos impactos da dor lombar em gestantes e as intervenções terapêuticas utilizadas. A busca eletrônica foi realizada entre junho e julho de 2021 nas bases de dados: PUBMED, LILACS, SCIELO e PEDro. Foram selecionados artigos dentro dos últimos 5 anos, nos idiomas inglês e português, originais, que estivessem disponíveis na íntegra de maneira gratuita, e publicados em periódicos indexados. A dor lombar possui causa multifatorial que limita significativamente diversas atividades de vida diárias de mulheres grávidas. Sua prevalência dentre os estudos foi alta, com destaque para grávidas multíparas, com histórico de dor lombar em gestações anteriores e lombalgia prévia à gravidez. As intervenções terapêuticas encontradas nos artigos foram o pilates, o kinesio taping, a acupuntura e principalmente exercícios físicos de intensidade baixa a moderada. A partir dos estudos identificados nesta revisão foi possível observar que a dor lombar afeta uma parcela significativa de mulheres durante a gestação, que limita as atividades de vida diária e diminui a capacidade funcional e qualidade de vida. De maneira geral, a implementação de atividades físicas e métodos terapêuticos não medicamentosos apresentam-se como possibilidades eficazes de intervenção para esse público com melhora dos sintomas e do bem estar dessas mulheres.

\footnotetext{
Abstract

The gestational period is marked by several morphological, biomechanical and physiological changes, despite being considered a normal event, several challenges are presented to pregnant women, especially low back pain. This study is an integrative literature review that seeks to survey the impacts of low back pain on pregnant women and the therapeutic interventions used. The electronic search was carried out between June and July 2021 in the following databases: PUBMED, LILACS, SCIELO and PEDro. Articles were selected within the last 5 years, in English and Portuguese, originals, which were available in full free of charge, and published in indexed journals. Low back pain has a multifactorial cause that significantly limits several daily activities of pregnant women. Its prevalence among the studies was high, with emphasis on multiparous pregnant women, with a history of low back pain in previous pregnancies and low back pain prior to pregnancy. The therapeutic interventions found in the articles were pilates, kinesio taping, acupuncture and especially low to moderate intensity physical exercises. From the studies identified in this review, it was possible to observe that low back pain affects a significant portion of women during pregnancy, which limits activities of daily living and decreases functional capacity and quality of life. In general, the implementation of physical activities and non-pharmacological therapeutic methods are presented as effective possibilities of intervention for this public, with improvement in symptoms and in the well-being of these women.
} 


\section{INTRODUÇÃO}

O período gestacional é marcado por diversas mudanças morfológicas, biomecânicas e fisiológicas, apesar de ser considerado um evento normal diversos desafios são apresentados a gestante. Aliado a isto, a redução significativa da mortalidade materna, acabou por enfatizar as morbidades presentes nesse período (ACHARYA et al., 2019).

Durante a gravidez a modificação na massa corporal e posicionamento dos órgãos, acaba por gerar uma mudança do centro de gravidade causando um aumento dos quadros álgicos, além de alterações na saúde mental que colaboram para a redução da frequência das realizações de atividades físicas (O'CONNOR et al., 2018; BRYNDAL et al., 2020).

Dentre as regiões mais acometidas têm-se a coluna vertebral em áreas como cervical, torácica e lombar sendo a última a mais prevalente, podendo ocorrer ainda dor por toda extensão da coluna, sendo mais comum o aparecimento nas regiões lombo sacra e dorso-lombar (MARTINS E SILVA, 2005).

No entanto, de maneira prevalente, a lombalgia se apresenta como problemática pois perdura desde o início da gestação, com um avanço progressivo no decorrer dos trimestres gravídicos (BACKHAUSEN et al., 2017). Estima-se que pelo menos 50\% das mulheres grávidas sentem dor lombar e esses desconfortos têm ligação com as transformações fisiológicas provisórias ocorridas no sistema musculoesquelético (MANYOZO et al., 2019).

Este quadro torna-se preocupante e merece atenção especial ao passo que a dor lombo pélvica apresenta-se como a causa mais frequente de licença médica entre as mulheres grávidas, além de limitar atividades que envolvam levantamento de peso, e realização de atividades diárias, trazendo dificuldades para caminhar, realizar tarefas como limpeza, trabalho e vida sexual (STAFNE et al., 2019).
Diante disto, esse estudo teve como objetivo realizar um levantamento da literatura acerca dos impactos da dor lombar em gestantes e as intervenções terapêuticas utilizadas.

\section{MATERIAL E METODOS}

Esse estudo trata-se de uma revisão integrativa da literatura. Para o levantamento, seguiu-se o seguinte caminho metodológico: primeiro definiu-se o problema a ser estudado a partir do questionamento sobre o impacto da dor lombar em gestantes e partindo da seguinte pergunta norteadora: qual o impacto da dor lombar em gestantes e qual e quais principais terapêuticas utilizadas para o tratamento? Em seguida foi realizada a seleção da amostra na literatura, seguida da caracterização dos artigos e análise crítica dos resultados incluídos, para discussão.

A busca eletrônica foi realizada entre junho e julho de 2021 nas bases de dados: PUBMED, LILACS, SCIELO e PEDro. Foram selecionados artigos que tinham sido publicados nos últimos 5 anos, nos idiomas inglês e português, artigos originais, que estivessem disponíveis na íntegra de maneira gratuita, e publicados em periódicos indexados. Tanto os estudos observacionais, como experimentais foram incluídos.

Foram excluídos artigos de revisão bibliográfica, editoriais, cartas aos editores, tese, dissertações e monografia. Aqueles artigos que tratavam sobre dores de forma geral ou que não especificaram sua classificação voltados para gestantes, também foram excluídos.

Para a pesquisa dos estudos, foram utilizadas as combinações de descritores através dos operadores booleanos AND e OR: pregnancy OR pregnants AND physical therapy AND low back pain AND Quality of Life sendo em português: gravidez OR gestação OR gestantes AND fisioterapia AND dor lombar AND qualidade de vida. 
Seguiu-se o critério de seleção na seguinte ordem: por título, seguida pela leitura do resumo e leitura completa para aqueles selecionados nas etapas anteriores.

\section{RESULTADOS}

Após a busca nas bases de dados, utilizando os descritores, foram encontrados 52 estudos, porém, apenas 15 artigos se encaixaram nos critérios de inclusão e foram usados no artigo. Desses 15 artigos incluídos no estudo, 11 foram encontrados na base de dados PuBmeD, 2 na LILACS, 1 na ScieLO e 1 na PEDro. A tabela 1 traz a descrição destes estudos selecionados.

De maneira geral, as características dos artigos apontam para uma maior publicação na língua inglesa. Com relação aos instrumentos utilizados para avaliação, aqueles que se destacaram foram: o questionário Roland Morris Disability ( $\mathrm{n}=5)$, a Escala Visual Analógica $(n=4)$ e o questionário Oswestry Low Back $(n=3)$.

O número amostral encontrado nos artigos variou de 20 à 1510 gestantes; e os anos de mais publicações foram 2019, 2017 e 2020 respectivamente. Com relação ao delineamento, foi observado um maior número de estudos randomizado controlado e de coorte transversal.

As intervenções terapêuticas encontradas nos artigos foram o pilates, o kinesio taping, a acupuntura e principalmente exercícios físicos de intensidade baixa a moderada. Em relação a prevalência de dor lombar, foi observada alta frequência em grávidas multíparas com histórico de dor lombar em gestações anteriores e lombalgia prévia à gravidez.

\section{DISCUSSÃO}

Algumas grávidas relatam que durante esse período têm dificuldades em realizar suas atividades de vida diária, o que afeta consideravelmente a sua qualidade de vida. Dentre os motivos, a dor lombar se apresenta como uma das complicações mais relatadas no período gravídico, afetando uma grande parcela das mulheres. Estudos demonstram ainda, que a dor geralmente surge no segundo trimestre e tende a piorar com a chegada do terceiro trimestre (SANTOS, 2018).

Estes números despertam uma relevante atenção clínica, pois a dor lombar é uma causa multifatorial que limita significativamente diversas atividades de vida diárias de mulheres grávidas. Um estudo realizado com 1.510 gestantes internadas em um hospital em Poznan, na Polônia, identificou por meio de um questionário alguns fatores que aumentam a intensidade da dor em grávidas. Dentre as participantes 49,6\% afirmaram que a dor aumenta na hora de dormir, $38,7 \%$ ao sentar-se, $37,2 \%$ ao caminhar e os outras apontaram o ato de curvar-se, ficar em pé, levantar objetos pesados e realizar atividades físicas (BRYNDAL et al., 2020). $\mathrm{Na}$ mesma linha de pensamento, Carvalho et al. (2017) relataram que a intensidade da dor aumenta à noite e que ficar em pé por longos períodos foi identificado como um dos principais motivos de agravo da dor na coluna.

Acharya et al. (2019), realizaram uma pesquisa com 1.284 grávidas e verificaram que existe uma maior probabilidade de se ter lombalgia relacionada a gravidez quando a mulher tem um índice de massa corporal (IMC) alto, aumento das semanas de gestação, maior nível de escolaridade e aquelas em que o marido tem ensino superior e maior renda mensal, uma vez que provavelmente ele trabalhando fora, a mulher realizará as atividades domesticas sozinha, resultando em mais esforços físicos e consequentemente no aumento das dores. 
Tabela 1. Características Gerais dos Artigos

\begin{tabular}{|c|c|c|c|c|c|}
\hline Autor & Título & Ano & Periódico & Objetivo & Metodologia \\
\hline $\begin{array}{l}\text { BRYNDAL et } \\
\text { al. }\end{array}$ & $\begin{array}{l}\text { Fatores de risco associados à } \\
\text { dor lombar entre um grupo de } \\
1510 \text { mulheres grávidas }\end{array}$ & 2020 & $\begin{array}{l}\text { Journal of } \\
\text { Personalized } \\
\text { Medicine }\end{array}$ & $\begin{array}{l}\text { Determinar os fatores de risco } \\
\text { associados à lombalgia } \\
\text { mulheres grávidas }\end{array}$ & $\begin{array}{l}\text { A amostra foi composta por } 1510 \text { gestantes, foi } \\
\text { utilizado um questionário sociodemográfico, a Escala } \\
\text { Visual Analógica (EVA) para avaliar a intensidade da } \\
\text { dor, e o questionário Roland Morris Disability para } \\
\text { avaliar o impacto da lombalgia na qualidade de vida } \\
\text { da gestante }\end{array}$ \\
\hline
\end{tabular}

CARVALHO et Efeitos da estabilização lombar $2020 \quad$ European

al.

e alongament dor, incapacidade, controle postural e ativação muscular em gestantes com lombalgia
Journal

Physical

Rehabilitation

Medicine
Comparar os efeitos dos exercícios Estudo piloto randomizado composto por 20 gestantes of de estabilização lombar e com lombalgia, as participantes foram randomizadas em and alongamento no tratamento da dois grupos de intervenção: o protocolo de exercícios de estabilização lombar e o protocolo de exercícios de alongamento, foi utilizado também a Escala Visual Analógica, o questionário de Dor de McGill e o questionário de incapacidade de Roland Morris

\begin{tabular}{|c|c|c|c|c|c|}
\hline $\begin{array}{l}\text { SÖNMEZER } \\
\text {,ÖZKÖSLÜ e } \\
\text { YOSMAOĞLU }\end{array}$ & $\begin{array}{l}\text { Os efeitos dos exercícios } \\
\text { clínicos de pilates na } \\
\text { incapacidade funcional, dor, } \\
\text { qualidade de vida e } \\
\text { estabilização lombo-pélvica em } \\
\text { mulheres grávidas com dor } \\
\text { lombar: um estudo controlado } \\
\text { randomizado }\end{array}$ & 2020 & $\begin{array}{l}\text { Journal of Back } \\
\text { and } \\
\text { Musculoskeletal } \\
\text { Rehabilitation }\end{array}$ & $\begin{array}{l}\text { Determinar os efeitos dos } \\
\text { exercícios clínicos de Pilates na } \\
\text { estabilização lombopélvica, dor, } \\
\text { incapacidade e qualidade de vida } \\
\text { em mulheres grávidas com } \\
\text { lombalgia }\end{array}$ & $\begin{array}{l}\text { Estudo duplo cego, randomizado e controlado } \\
\text { composto por } 40 \text { gestantes divididas em dois grupos: } \\
\text { grupo de exercícios de pilates e grupo controle, foi } \\
\text { utilizado a Escala Visual Analógica, o questionário } \\
\text { Oswestry Low Back Pain e o Nottingham Health Profile } \\
\text { para avaliar dor, incapacidade e qualidade de vida } \\
\text { respectivamente }\end{array}$ \\
\hline $\begin{array}{l}\text { ACHARYA } \\
\text { al. }\end{array}$ & $\begin{array}{l}\text { Prevalência e gravidade da } \\
\text { região lombar e dor na cintura } \\
\text { pélvica mulheres em grávida } \\
\text { nepalesa }\end{array}$ & 2019 & $\begin{array}{l}\text { BMC Pregnancy } \\
\text { and Childbirth }\end{array}$ & $\begin{array}{l}\text { Avaliar a prevalência e a gravidade } \\
\text { da lombalgia e/ ou dor na cintura } \\
\text { pélvica entre mulheres grávidas } \\
\text { nepalesas, bem como explora os } \\
\text { fatores associados à lombalgia e à } \\
\text { dor na cintura pélvica }\end{array}$ & $\begin{array}{l}\text { Estudo transversal com gestantes recrutadas em dois } \\
\text { hospitais do distrito de Nepal, foi utilizado um } \\
\text { questionário sobre características sociodemográficas, } \\
\text { gestacionais e de carga de trabalho para avaliar a } \\
\text { prevalência da dor lombar }\end{array}$ \\
\hline $\begin{array}{l}\text { MANYOZO et } \\
\text { al. }\end{array}$ & $\begin{array}{l}\text { Dor lombar durante a gravidez: } \\
\text { prevalência, fatores de risco e } \\
\text { associação com atividades } \\
\text { diárias entre mulheres grávidas } \\
\text { na área urbana de Blantyre - } \\
\text { Malawi }\end{array}$ & 2019 & $\begin{array}{l}\text { Malawi Medical } \\
\text { Journal }\end{array}$ & $\begin{array}{l}\text { Investigar a prevalência e os fatores } \\
\text { de risco da lombalgia e sua } \\
\text { associação com atividades } \\
\text { funcionais em mulheres grávidas } \\
\text { no Malaui }\end{array}$ & $\begin{array}{l}\text { Estudo transversal quantitativo, realizado em } 5 \text { unidades } \\
\text { de saúde locais na área urbana de Blantyre, Malawi, foi } \\
\text { utilizado o questionário desenvolvido pelos } \\
\text { pesquisadores para avaliar a prevalência de lombalgia e o } \\
\text { questionário Oswestry Low Back Pain Disability para } \\
\text { coletar dados sobre o estado funcional das gestantes }\end{array}$ \\
\hline
\end{tabular}




\begin{tabular}{|c|c|c|c|c|c|}
\hline $\begin{array}{l}\text { NICOLIAN et } \\
\text { al. }\end{array}$ & $\begin{array}{l}\text { Custo Efetividade da } \\
\text { acupuntura versus tratamento } \\
\text { padrão para dor pélvica e } \\
\text { lombar na gravidez: um ensaio } \\
\text { clínico randomizado }\end{array}$ & 2019 & Plos One & $\begin{array}{l}\text { Avaliar o custo efetividade da } \\
\text { acupuntura para dor lombar } \\
\text { durante a gravidez }\end{array}$ & $\begin{array}{l}\text { Estudo clínico randomizado e pragmático, composto } \\
\text { por gestantes de cinco maternidades da aérea de Paris, as } \\
\text { participantes foram randomizadas em paralelo para } \\
\text { receber tratamento padrão ou tratamento padrão mais } \\
\text { acupuntura }\end{array}$ \\
\hline $\begin{array}{l}\text { O'CONNOR et } \\
\text { al. }\end{array}$ & $\begin{array}{l}\text { Efeitos do treinamento de } \\
\text { resistência em domínios } \\
\text { relacionados à fadiga de } \\
\text { qualidade de vida e humor } \\
\text { durante a gravidez: um ensaio } \\
\text { randomizado em mulheres } \\
\text { grávidas com dor nas costas }\end{array}$ & 2019 & $\begin{array}{l}\text { Journal of } \\
\text { Biobehavioral } \\
\text { Medicine- } \\
\text { Psychosomatic } \\
\text { Medicine }\end{array}$ & $\begin{array}{l}\text { Verificar se o treinamento de } \\
\text { resistência de intensidade baixa a } \\
\text { moderada durante as semanas } 22- \\
34 \text { de gravidez pode melhorar a } \\
\text { qualidade de vida e o humor }\end{array}$ & $\begin{array}{l}\text { Estudo controlado randomizado de grupo paralelo com } \\
\text { gestantes do } 2 \text { trimestre com dor nas costas ou histórico } \\
\text { de dor nas costas, para realizarem treinamento de } \\
\text { resistência de intensidade baixa a moderada duas vezes } \\
\text { por semana, a qualidade de vida e o humor foram } \\
\text { medidos antes e depois das intervenções usando o Short } \\
\text { Form Health Survey e Profile of Mood States }\end{array}$ \\
\hline STAFNE et al. & $\begin{array}{l}\text { Impacto da adaptação ao } \\
\text { trabalho, localização da dor e } \\
\text { exercícios na licença médica } \\
\text { devido à dor lombo pélvica na } \\
\text { gravidez: um estudo } \\
\text { longitudinal }\end{array}$ & 2019 & $\begin{array}{l}\text { Scandinavian } \\
\text { Journal of } \\
\text { Primary Health } \\
\text { Care }\end{array}$ & $\begin{array}{l}\text { Identificar os fatores associados ao } \\
\text { afastamento por doença } \\
\text { lombopélvica (LPP) na gestação }\end{array}$ & $\begin{array}{l}\text { Estudo de coorte prospectivo, composto por } 2 \text { grupos, } \\
\text { onde um grupo de grávidas foram submetida s ao } \\
\text { programa de exercícios e outro grupo foi feita } \\
\text { atendimento pré natal padrão, para analisar o desfecho } \\
\text { desses grupos na licença médica devido a LPP no final } \\
\text { da gravidez }\end{array}$ \\
\hline VAS et al. & $\begin{array}{l}\text { Efeito da acupuntura auricular } \\
\text { na dor relacionada à gravidez } \\
\text { na região lombar e na cintura } \\
\text { pélvica posterior: um ensaio } \\
\text { clínico multicêntrico } \\
\text { randomizado }\end{array}$ & 2019 & $\begin{array}{l}\text { Acta Obstetricia } \\
\text { et Gynecologica } \\
\text { Scandinavica }\end{array}$ & $\begin{array}{l}\text { Avaliar o efeito da acupuntura } \\
\text { auricular associada aos cuidados } \\
\text { obstétricos padrão, no ambiente de } \\
\text { atenção primária, na dor da região } \\
\text { lombar e / ou na cintura pélvica } \\
\text { posterior } \\
\text { vivenciada por mulheres grávidas }\end{array}$ & $\begin{array}{l}\text { Estudo clínico controlado randomizado multicêntrico de } \\
\text { quatro grupos, composto por } 220 \text { gestantes que foram } \\
\text { aleatoriamente designados para receber cuidados } \\
\text { obstétricos padrão mais duas sessões de acupuntura de } \\
\text { ouvido, ou acupuntura de ouvido não específica, ou } \\
\text { acupuntura de ouvido com placebo ou apenas cuidados } \\
\text { obstétricos padrão }\end{array}$ \\
\hline SANTOS et al. & $\begin{array}{l}\text { Estudo comparativo da } \\
\text { influência da lombalgia nas } \\
\text { atividades de vida diária entre } \\
\text { primigestas e multíparas }\end{array}$ & 2018 & $\begin{array}{l}\text { Fisioterapia } \\
\text { Brasil }\end{array}$ & $\begin{array}{l}\text { Avaliar a classificação do nível de } \\
\text { incapacidade da lombalgia em } \\
\text { gestantes primigestas e multíparas }\end{array}$ & $\begin{array}{l}\text { Estudo observacional descritivo de corte transversal, } \\
\text { composto por } 50 \text { gestantes com idade entre } 18 \text { a } 30 \text { anos, } \\
\text { foi aplicado um questionário para saber o IMC, histórico } \\
\text { gestacional e histórico de dor lombar, e o Questionário } \\
\text { de Oswestry de Desabilidade de Dor Lombar para } \\
\text { investigar a interferência da lombalgia nas atividades } \\
\text { diárias }\end{array}$ \\
\hline $\begin{array}{l}\text { BACKHAUS } \\
\text { EN et al. }\end{array}$ & $\begin{array}{l}\text { Os efeitos de um programa de } \\
\text { exercícios aquáticos não } \\
\text { supervisionado na dor lombar e } \\
\text { licença médica entre mulheres } \\
\text { grávidas saudáveis - Um ensaio }\end{array}$ & 2017 & Plos One & $\begin{array}{l}\text { Avaliar o efeito do programa de } \\
\text { exercícios aquáticos não } \\
\text { supervisionado na intensidade da } \\
\text { dor lombar e dias passados em } \\
\text { licença médica entre mulheres }\end{array}$ & $\begin{array}{l}\text { Estudo randomizado controlado de grupos paralelos, } \\
\text { com } 516 \text { gestantes que foram aleatoriamente designadas } \\
\text { para exercícios aquáticos não supervisionados duas vezes } \\
\text { por semana por um período de } 12 \text { semanas ou } \\
\text { atendimento pré natal padrão, os desfechos foram }\end{array}$ \\
\hline
\end{tabular}




\begin{tabular}{|c|c|c|c|c|c|}
\hline & randomizado controlado & & & grávidas saudáveis & $\begin{array}{l}\text { analisados pela escala Low Back Pain Rating, } \\
\text { questionário Roland Morris Disability e pela } \\
\text { autoavaliação de saúde geral }\end{array}$ \\
\hline $\begin{array}{l}\text { CARVALHO et } \\
\text { al. }\end{array}$ & Lombalgia na gestação & 2017 & $\begin{array}{l}\text { Revista } \\
\text { Brasileira de } \\
\text { Anestesiologia }\end{array}$ & $\begin{array}{l}\text { Avaliar a frequência da lombalgia e } \\
\text { suas características na gestação }\end{array}$ & $\begin{array}{l}\text { Estudo de coorte transversal com gestantes de baixo } \\
\text { risco do primeiro ao terceiro trimestre gestacional, foi } \\
\text { aplicado o questionário com perguntas como a idade, } \\
\text { peso e profissão, informações relacionadas à gestação e } \\
\text { à presença ou não de lombalgia }\end{array}$ \\
\hline $\begin{array}{l}\text { KALINOWSKI } \\
\text { e } \\
\text { KRAWULSKA }\end{array}$ & $\begin{array}{l}\text { Kinesio Taping vs. Placebo na } \\
\text { redução da dor lombar } \\
\text { relacionada à gravidez: Um } \\
\text { estudo cruzado }\end{array}$ & 2017 & $\begin{array}{l}\text { Medical Science } \\
\text { Monitor }\end{array}$ & $\begin{array}{l}\text { Determinar a influência do Kinesio } \\
\text { Taping na redução da dor lombar } \\
\text { em mulheres }\end{array}$ & $\begin{array}{l}\text { Estudo experimental randomizado e cego, composto } \\
\text { por } 106 \text { gestantes que foram divididos aleatoriamente } \\
\text { em } 2 \text { grupos: fitas Kinesio Tex e placebo, foi utilizado a } \\
\text { Escala Visual Analógica e o questionário Roland Morris } \\
\text { Disability para avaliar a dor }\end{array}$ \\
\hline LIMA et al. & $\begin{array}{l}\text { Prevalência de lombalgia e a } \\
\text { interferência na qualidade de } \\
\text { vida de gestantes }\end{array}$ & 2017 & Revista Dor & $\begin{array}{l}\text { Determinar a prevalência de } \\
\text { lombalgia e a interferência na } \\
\text { qualidade de vida de gestantes } \\
\text { atendidas na Estratégia de Saúde da } \\
\text { Família da Cidade de Cabo Frio }\end{array}$ & $\begin{array}{l}\text { Estudo de campo transversal, composto por mulheres } \\
\text { em seu período gestacional assistidas pelo programa de } \\
\text { atendimento do pré-natal de baixo risco, foi utilizado o } \\
\text { questionário sociodemográfico, o questionário Roland } \\
\text { Morris Disability para avaliar a incapacidade funcional da } \\
\text { gestante com lombalgia e o questionário WHOQOL- } \\
\text { bref }\end{array}$ \\
\hline BISHOP et al. & $\begin{array}{l}\text { Avaliando a acupuntura e o } \\
\text { tratamento padrão para } \\
\text { mulheres grávidas com dor nas } \\
\text { costas: o estudo piloto } \\
\text { randomizado } \quad \text { controlado } \\
\text { EASE Back }\end{array}$ & 2016 & $\begin{array}{l}\text { National } \\
\text { Institute for } \\
\text { Health Research }\end{array}$ & $\begin{array}{l}\text { Avaliar o benefício da acupuntura } \\
\text { para dor nas costas relacionada à } \\
\text { gravidez }\end{array}$ & $\begin{array}{l}\text { Estudo piloto randomizado controlado, realizado com } \\
\text { gestantes que relataram ter lombalgia, para avaliar o } \\
\text { efeito dos tratamentos sobre a dor }\end{array}$ \\
\hline
\end{tabular}


Para Bryndal et al. (2020) e Kalinowisk e krawulska (2017), a mulher também apresenta uma maior chance de ter fortes dores na lombar quando a gravidez é múltipla e em cada nova gestação, pois se durante a primeira gravidez a mulher sentiu dor na lombar, ela irá experimentar dores recorrentes em gestações seguintes.

De acordo com Bishop et al. (2016), a dor lombar aumenta com o avançar da gravidez e geralmente é mais forte no período noturno atrapalhando o sono. Carvalho et al. (2017) fizeram entrevistas com 97 gestantes onde $68 \%$ delas relataram sentir dores na lombar que iniciaram no segundo trimestre da gravidez e dessas, $71,2 \%$ relataram que o desconforto é mais frequente a noite o que prejudica seu sono e sua vitalidade do dia seguinte.

Apesar de ser um problema recorrente entre as grávidas, o tratamento ainda é pouco realizado entre elas. Isso se dá ao fato de a dor lombar ainda não ser vista como uma complicação grave nesse período, já que é um sintoma comum (ACHARYA et al., 2019). Isto se apresenta como uma problemática, ao passo que essa falta de cuidado e atenção pode afetar negativamente a capacidade da mulher de conseguir andar, sentar e fazer suas atividades diárias, o que impacta negativamente a sua vida de maneira geral e suas relações familiares e sociais (MANYOZO et al., 2019).

Considerando todos os problemas mencionados, é importante que a mulher grávida procure métodos que a ajudem a aliviar a dor na coluna lombar. A literatura traz diversas abordagens que podem ser utilizadas com ajuda de um profissional capacitado, para a melhora do quadro, como atividades físicas, caminhadas, natação, exercícios de respiração e terapia manual, que melhoram a capacidade funcional (KALINOWISK e KRAWULSK, 2017).
Dentre as intervenções possíveis, Sonmezer et al. (2020), em seu estudo com 40 mulheres grávidas, mostrou a eficácia do pilates na melhora da dor lombar. As participantes foram divididas em dois grupos, as que fizeram os exercícios de pilates e outras 20 ficaram no grupo controle. Os resultados da abordagem demonstraram eficácia maior naquelas mulheres que fizeram os exercícios, demonstrando aumento da força, redução da dor e da incapacidade, além da melhora do sono e da mobilidade física das gravidas.

Para Backhausen et al. (2017), no período gestacional é importante que a mulher tenha hábitos de praticar exercícios físicos por pelo menos 30 minutos ao dia, pois essas atividades ajudam a diminuir os riscos de dor lombar. Isto foi evidenciado por meio do seu estudo com 516 grávidas saudáveis submetidas a exercícios aquáticos, durante 12 semanas, sendo que aquelas participantes que ficaram no grupo intervenção apresentou uma melhora da dor lombar, além de que foi visto que a natação é uma abordagem eficaz e segura na gravidez.

Outro método que desponta como possibilidade para esse período é o kinesio taping, uma abordagem terapêutica usada para redução das tensões e do quadro álgico na região da lombar. Um estudo incluiu 106 mulheres no segundo e terceiro trimestre da gravidez, com lombalgia, onde as participantes foram divididas em dois grupos, as que receberam a aplicação do método e as que utilizaram placebo. Ao final da pesquisa foi identificado que a dor lombar em grávidas diminuiu significativamente após o uso da bandagem dinâmica, quando comparado ao placebo (KALINOWSKI e KRAWULSKA, 2017).

O'Connor et al. (2018), recrutaram 134 mulheres grávidas para receberem aulas de educação para gravidez e treinamento de resistência com intensidade baixa a moderada. As participantes foram acompanhadas por especialistas e a intervenção durou 
cerca de 12 semanas. As mulheres que realizaram o treinamento relataram melhora na vitalidade e houve uma diminuição na sensação de fadiga, trazendo maior conforto e melhora na qualidade de vida.

Tais abordagens como visto na literatura, trazem inúmeros benefícios para a gestante. Nesse sentido, é importante que as mulheres busquem métodos para a melhora da dor, pois dependendo do grau a dor na lombar pode perdurar após a gestação. Então, iniciar atividades físicas neste período trará benefícios e proporcionará uma gravidez mais ativa e saudável (KALINOWSKI e KRAWULSKA, 2017).

\section{CONCLUSÕES}

A partir dos estudos identificados nesta revisão foi possível observar que a dor lombar possui causa multifatorial que afeta uma parcela significativa de mulheres durante a gestação, o que limita diversas atividades de vida diária e consequentemente diminuindo sua capacidade funcional e qualidade de vida. Apesar da problemática recorrente entre as gravidas, ainda existe a negligência na realização do tratamento.

O pilates, kinesio taping e o treino de resistência com intensidade baixa a moderada foram os recursos evidenciados no estudo e que trouxeram benefícios para o quadro álgico e funcional das gestantes, tais como: aumento da força, redução da dor e da incapacidade, melhora do sono e da mobilidade física, redução das tensões, diminuição na sensação de fadiga, proporcionando maior conforto e melhora da qualidade de vida. Sendo assim, torna-se possível incentivar a implementação de atividades físicas e métodos terapêuticos não medicamentosos, nesse público-alvo, de modo a favorecer o bem-estar nesse período.

\section{REFERÊNCIAS}

Acharya RS, Stuge B, Tveter TA, Grotle M, EberhardGran. Prevalence and severity of low back- and pelvic girdle pain in pregnant Nepalese women. BMC Pregnancy and Childbirth. 2019;19:2-11. doi:10.1186/s12884-019-2398-0

Backhausen MG, Tabor A, Albert H, Rosthøj S, Damm $\mathrm{P}$, Hegaard HK. The effects of an unsupervised water exercise program on low back pain and sick leave among healthy pregnant women - A randomised controlled trial. PLoS ONE. 2017;12:1-16. doi:10.1371/journal.pone.0182114

Bishop A, Ogollah R, Bartlam B, Barlas P, Holden MA, Ismail KM, Jowett S, Lewis M, Lloyd A, Kettle $\mathrm{C}$ et al. Assessing Acupuncture and Standard Treatment for pregnant women with back pain: o randomized controlled pilot study EASE Back. American journal of obstetrics and gynecology. 2016;2:2-19. doi:10.1186/s40814-016-0107-6

Bryndal A, Majchzycki M, Grochulska A, Glowinski S, Seremak-Mrozikiewicz A. Risk Factors Associated with Low Back Pain among A Group of 1510 Pregnant Women. J. Pers. Med. 2020;10:1-7. doi:10.3390/jpm10020051

Carvalho APF, Dufresne SS, Oliveira MR, Furlanetto CK, Dubois M, Dallaire et al. Effects of lumbar stabilization and muscular stretching on pain, disabilities, postural control and muscle activation in pregnant woman with low back pain. European journal of physical and rehabilitation medicine. 2020;56:297-306. doi:10.23736/S1973-9087.20.06086-4

Carvalho MECC, Lima LC, Terceiro CAL, Pinto DRL, Silva MN, Cozer GA et al. Low back pain during pregnancy. Rev Bras Anestesiol. 2017;67:266270. doi: 10.1016/j.bjan.2016.03.002

Kalinowski P, Krawulska A. Kinesio Taping vs. Placebo in Reducing Pregnancy-Related Low Back Pain: A Cross-Over Study. Med Sci Monit. 2017;26:6114-6120. doi: 10.12659/msm.904766

Lima ACN, Oliveira FB, Avolio GP, Silva GD, Silva PS, Vale RGS. Prevalence of low back pain and interference with quality of life of pregnant women. Rev Dor. 2017;18:119-23. doi:10.5935/1806-0013.20170024

Manyozo SD, Nesto T, Bonongwe P, Muula AS. Low back pain during pregnancy: Prevalence, risk 
factors and association with daily activities among pregnant women in urban Blantyre, Malawi. Malawi Medical Journal. 2019;31:71-76. doi:10.4314/mmj.v31i1.12

Martins RF, Silva JLP. Prevalência de dor nas costas na gestação. Rev Assoc Med Bras 2005;51:1447144.

Nicolian S, Butel T, Gambotti L, Durand M, FilipovicPierucci A, Mallet A, et al. Custo-efetividade da acupuntura versus tratamento padrão para dor pélvica e lombar na gravidez: um ensaio clínico randomizado. PLoS ONE. 2019;14:1-16. doi:10.1371/journal. pone.0214195

O’Connor PJ, Poudevigne MS, Johnson KE, Araujo JB, Ward-Ritacco CL. Effects of resistance training on fatigue-related domains of quality of life and mood during pregnancy: A randomized trial in pregnant women with back pain. PsychosomMed. 2018;80:327-332. doi:10.1097/PSY.0000000000000559

Santos DH, Cunha CMP, Lobo RP, Andrade MA, Nepomuceno EJ, Maia JN. Estudo comparativo da influência da lombalgia nas atividades de vida diária entre primigestas e multíparas. Fisioter Bras. 2018;19:171-176 doi:10.33233/fb.v19i2.2305

Sonmezer E, Özköslü MA, Yosmaoğlu HB. The effects of clinical pilates exercises on functional disability, pain, quality of life and lumbopelvic stabilization in pregnant women with low back pain: A randomized controlled study. J Back Musculoskelet Rehabil. 2021;34:69-76. doi:10.3233/BMR-191810

Stafne SN, Vøllestad NK, Mørkved S, Salvesen KA, Robinson HS. Impact of job adjustment, pain location and exercise on sick leave due to lumbopelvic pain in pregnancy: a longitudinal study. Scandinavian journal of primary health care. 2019;37:218-226. doi:10.1080/02813432.2019.1608058

Vas J, Cintado MC, Aranda-Regules JM, Aguilar I, Rivas Ruiz F. Effect of ear acupuncture on pregnancy-related pain in the lower back and posterior pelvic girdle: A multicenter randomized clinical trial. Acta Obstet Gynecol Scand. 2019;98:1307-1317. doi: $10.1111 /$ aogs. 13635 